\title{
Design Considerations and Optimization of Calorimetric Flow Sensor for Respiratory Monitoring
}

\author{
Vasileios Kitsos ${ }^{1}$, Simeon West ${ }^{2}$, Andreas Demosthenous ${ }^{1}$, Xiao Liu ${ }^{1}$ \\ ${ }^{1}$ UCL Department of Electronic \& Electrical Engineering, London, WC1E 7JE, UK \\ ${ }^{2}$ University College Hospital, London, NW1 2BU, UK \\ Email:xiao@ucl.ac.uk
}

\begin{abstract}
This paper evaluates the impact of the operating modes, power consumption, and placement of temperature sensors against the heater to the design of a calorimetric flow sensor, for the range of 4.7 to 56.5 liters per minute (slm). In contrast to previous works most of which simply indicated the choice of various design parameters rather than providing a justification, this work provides useful guidelines for optimizing low-power small-area flow sensors for respiratory monitoring applications. A figure of merit (FoM) which is defined as the product of power consumption and sensor size, the two most challenging design parameters in developing small medical devices and systems, is proposed for quantifying flow sensor performance. Although the analysis and simulation was drawn upon designs in the $\mathbf{m m}$ scale, a similar optimization process can be applied to flow sensors of any size.
\end{abstract}

\section{INTRODUCTION}

Flow sensors are widely used in medicine for measuring respiratory flows. The purpose of their use vary from routing monitoring, such as for mechanically ventilated patients [1], to early diagnosis of certain diseases or medical complications, such as asthma [2].

Thanks to the rapid advance of microfabrication technology [3], thermal-type flow sensors are becoming increasingly prevalent in biomedical applications [4][5][6] where the physical size of the sensor is of great importance. Thermal flow sensors require no moving parts, can be made very small and offer high sensitivity to low flow rates. Among the most common topologies of thermal flow sensors in miniature medical devices or microsystems is the calorimetric type as it can be implemented in an easy and inexpensive way while it offers good accuracy as well as flow orientation information. Their operation is based on the temperature gradient near the central heater that arises when flow occurs. This gradient can be measured by two equally spaced temperature sensors at the upstream and downstream of the flow, respectively [7].

A flow sensor for respiratory monitoring applications needs to be as small as possible in order to minimize interference to flow. Any impeding to the flow may lead to turbulence, which, consequently, results in an increase in the flow resistance. It is important that any considerable increase in flow resistance, especially in cases of continuous respiration monitoring for patients with breathing difficulties, should be avoided. Also, the temperature rise due to the heater should be small enough if the sensor is placed close to human tissue so it does not cause any burn or discomfort to the patient or alter the property of the fluid.

Research supported by Doctoral Training Award from the UK Engineering and Physical Science Research Council.
Excessive power must also be avoided since it increases the thermal load that might lead to the sensor's breakdown.

The majority of previous and existing works on flow sensors limit themselves to one [8] or several of the operating modes, such as constant power and constant temperature. However, they gave no clear justification why a particular mode was chosen and provided no evidence for the chosen distance between the heater and the temperature sensors [9].

For miniature calorimetric flow sensors, the power consumption and the physical size of a flow sensor system are the two most important parameters that need to be optimized. The power consumption of a flow sensor system consists of the heater power and the power of the readout electronics. The latter is usually much smaller than the former, and the optimization of the power for readout electronics depends on the circuit design techniques and manufacturing. The size of the readout electronics (incl. temperature sensors, ADCs, etc.) can be made very small, should commercial purpose-made integrated circuits are used. Therefore, the size of a flow sensor is defined proportional to the distance between the heater and the temperature sensor.

In this paper, we compare calorimetric flow sensor performance under the three possible operating modes and provides guidelines on how to optimize sensor design towards a novel figure of merit which is defined as the product of heater power and sensor size.

\section{DIFFERENT OPERATING MODES}

King derived an experimental equation for the convective heat transfer from an infinite cylinder in an incompressible low Reynolds flow, that correlates the Nusselt with the Reynolds number, known as King's law [10]:

$$
N u=A^{\prime}+B^{\prime} \sqrt{R e}
$$

where $A$ ' and $B$ ' are constants that depend on the geometry and the materials of the flow sensor, $\mathrm{Nu}$ and $\mathrm{Re}$ are the Nusselt and Reynolds numbers, respectively, and defined as:

$$
\begin{gathered}
N u=\frac{h L}{k} \\
R e=\frac{\rho L}{\mu} U
\end{gathered}
$$

where $h$ is the heat transfer coefficient and $L$ is the characteristic length of the system. In addition, $\rho, k, \mu$ and $U$ are the fluid's density, thermal conductivity, viscosity and velocity, 


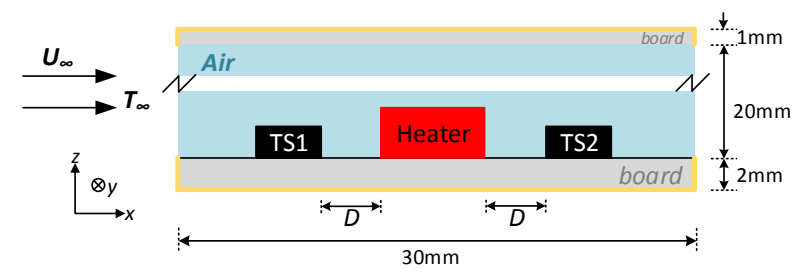

Figure 1. Illustration of the simulation setup. The temperature sensors TS1 and $T S 2$ are placed symmetrically to the heater at distance $D$. The yellow line highlights the thermal insulation points of the model.

respectively. According to the Newton's law of cooling, $h$ is related to the convective heat flux $q\left(\mathrm{~W} / \mathrm{m}^{2}\right)$ as:

$$
h=\frac{q}{T_{h o t}-T_{\infty}}
$$

where $T_{\text {hot }}$ is the temperature of the heater and $T_{\infty}$ is the inlet fluid temperature (assumed to be unaffected by the heater). Substituting (2), (3) and (4) into (1), it can be proven that:

$$
\frac{P}{\left(T_{h o t}-T_{\infty}\right)}=A+B \sqrt{U}
$$

where $P=q \cdot S$ is the power generated at the heater and $S$ is the surface area of the heater, $A$ and $B$ are the new constants that depend on the fluid, geometry and materials of the flow sensor. Based on (5), a flow sensor's heater can possibly work in one the following three operating modes: Constant Power (CP) where $P$ is constant; Constant Temperature (CT) where $T_{\text {hot }}$ is constant (and $P$ is varied); and Constant Temperature Difference (CTD) where $T_{\text {hot }}-T_{\infty}$ is constant (and $P$ is varied).

\section{Simulation SETUP}

The simulation model is shown in Fig. 1. A two-dimensional design of a flow sensor has been developed using the COMSOL Multiphysics ${ }^{\circledR}$ software, consisting of a heater with dimensions $3.15 \mathrm{~mm}$ (width) $\times 0.4 \mathrm{~mm}$ (height) placed on a printed circuit board (PCB) while a tunnel of $20 \mathrm{~mm}$ diameter is created over it. $U_{\infty}$ is the far-field, uniform velocity of the incoming air. Assuming that there is not any heat or velocity gradient in $y$ direction, a 2D model should be adequate to optimize the flow sensor. In addition, the flow within the tunnel is defined as laminar, starting developing from the edge of the board. The choice of a laminar flow is rather important since it defines the set of equations that describe the flow profile and the temperature distribution in the fluid. Also, it is assumed that the pressure drop between the inlet and outlet is negligible, thus, the flow is considered as incompressible and there is no work done due to zero pressure difference. Since the contribution of radiation towards heat transfer is practically insignificant when being compared to that of the forced convection, only the latter

TABLE I. PhysicAl Properties of MATERIALS USED

\begin{tabular}{|c|c|c|c|c|c|}
\hline \multicolumn{2}{|l|}{} & $\begin{array}{c}\text { Heater } \\
(\mathbf{N i})\end{array}$ & $\begin{array}{c}\text { Board } \\
(\boldsymbol{F R 4})\end{array}$ & $\begin{array}{c}\text { Air } \\
\left(35^{\circ} \mathrm{C}\right)\end{array}$ \\
\hline $\begin{array}{c}\text { Thermal } \\
\text { Conductivity }\end{array}$ & $k$ & $(\mathrm{~W} / \mathrm{m} \cdot \mathrm{K})$ & 11.3 & 0.3 & 0.027 \\
\hline Density & $\rho$ & $\left(\mathrm{kg} / \mathrm{m}^{3}\right)$ & 8400 & 1900 & 1.13 \\
\hline $\begin{array}{c}\text { Specific Heat } \\
\text { Capacity }\end{array}$ & $C_{\mathrm{P}}$ & $(\mathrm{J} / \mathrm{kg} \cdot \mathrm{K})$ & 450 & 1369 & 1007 \\
\hline $\begin{array}{c}\text { Dynamic } \\
\text { Viscosity }\end{array}$ & $\mu$ & $(\mathrm{kg} / \mathrm{m} \cdot \mathrm{s})$ & & & $1.9 \times 10^{-5}$ \\
\hline
\end{tabular}

is considered in the simulation which also makes the simulation faster. On the other hand, the main heat transfer phenomenon within the boards and heater, is conduction, described by the diffusion equation:

$$
\nabla^{2} T+\frac{q}{k}=\frac{1}{a} \frac{d T}{d t}
$$

where $q$ is the volumetric heat rate and $\alpha$ the thermal diffusivity of the material of interest. However, since it is a steady-state problem while heat is generated only in the heater, Eq. (6) can be simplified to the Laplace's equation $\nabla^{2} T=0$ and $\nabla^{2} T+$ $q / k=0$ for the boards and heater, respectively. The rest of the boundary conditions are the insulation at the external walls of the boards highlighted in Fig. 1, and the convection surface condition between the inner boards' walls and the fluid. The heat loss from the heater is either through the flow or to the substrate. The latter is dictated by the thermal conductivities of the board and heater and it can be minimized by choosing a board material with smaller $k$. The optimization of the heat loss through the substrate is beyond the scope of this paper as it would be specific to different board materials.

The different properties of various parts in the setup are listed in Table I. Furthermore, the relative humidity of the fluid is defined as $90 \%$ while the simulations are performed for two different $T_{\infty}, 20{ }^{\circ} \mathrm{C}$ and $35{ }^{\circ} \mathrm{C}$, in an effort to reproduce the environment of a medical flow sensor during respiration, such as that at the distal end of an intratracheal tube.

The output of the flow sensor is the temperature difference, $\Delta T\left(D, U_{\infty}\right)$, between the two temperature sensors (see TS1 and TS2 in Fig. 1) which are placed symmetrically on either side of the heater. For a defined velocity, a high temperature difference is important because i) it indicates the sensor is sensitive to the flow, hence able to measure small changes of flow and ii) it is then relatively easy to implement a temperature readout circuit. However, the high temperature difference may come at the expense of an elevated power consumption and increased sensor size. The simulated velocities are $0.25,1.25$ and $3 \mathrm{~m} / \mathrm{s}$, which correspond to 4.7, 23.6 and $56.5 \mathrm{slm}$ for the aforementioned setup. A respiratory rate of 12 to 18 breaths per minute is considered normal for healthy adults at rest [11]. Considering a tidal volume of $500 \mathrm{~mL}$, the flow rate of a healthy individual at rest can be approximated as 12 to $18 \mathrm{slm}$. Hence, the simulated velocities cover a variety of breathing conditions of different flow rates.

The initial conditions for all three modes are set as $5 \mathrm{~W}$ for $3 \mathrm{~m} / \mathrm{s}$ inlet velocity at $35^{\circ} \mathrm{C}$. As a result, the $\mathrm{CP}$ operates at $5 \mathrm{~W}$, the $\mathrm{CT}$ at $45.1^{\circ} \mathrm{C}$, and the $\mathrm{CTD}$ at $10.1^{\circ} \mathrm{C}$ overtemperature.

\section{Simulation RESUlts}

\section{A. Different Operating Modes}

Fig. 2 shows the simulation results for different operating modes. The first column shows the heater power vs. velocity (in bar graphs) and heater temperature vs. velocity (in line curves) for the CP, CT and CTD modes while the second column shows the temperature difference between the two temperature sensors when the distance between the temperature sensor and the heater is swept from 0 to $13.4 \mathrm{~mm}$. In the CP mode the $T_{\text {hot }}$ varies depending on the $T_{\infty}$ and velocity (Fig. 2a). It is also shown that for a defined distance $D$ from the heater, the output is 

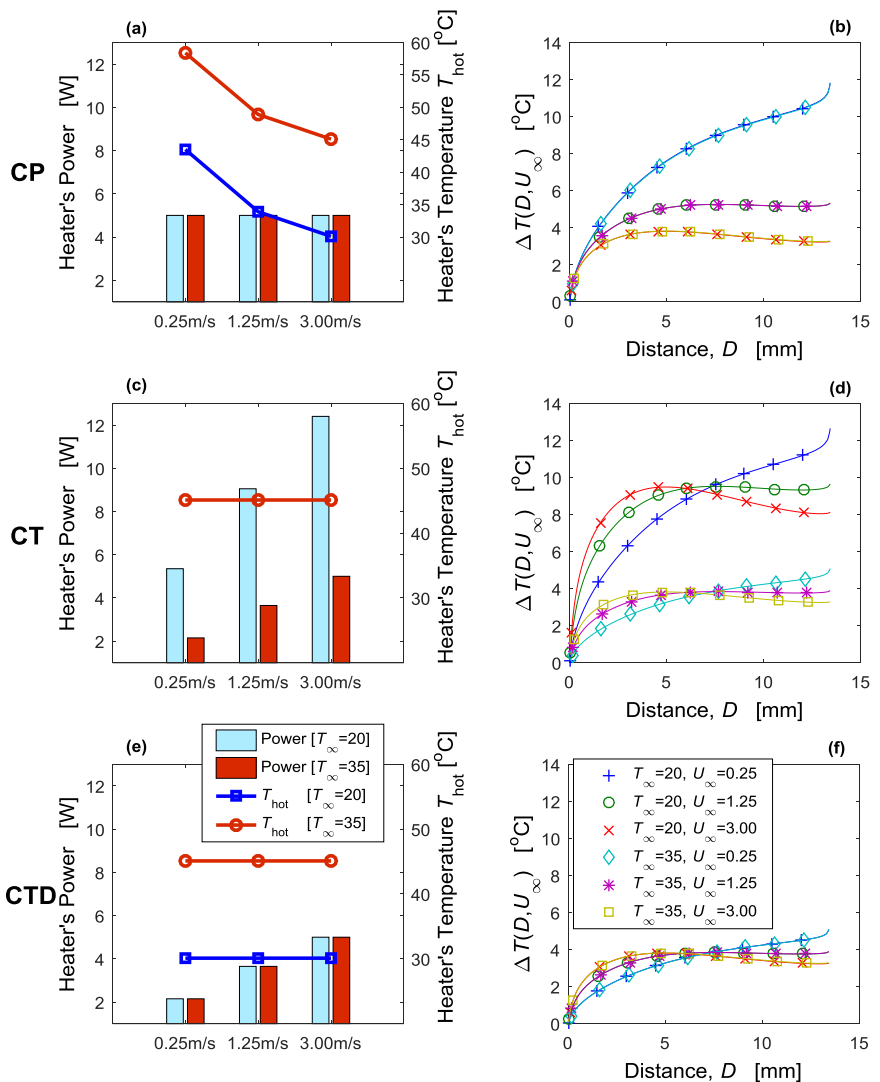

Figure 2. The CP, CT and CTD mode at the first, second and third row, respectively. The power consumption and the heater temperature are shown in the first column. The temperature difference versus the distance from the heater is indicated in the second column.

independent of $T_{\infty}$ (Fig. 2b). Hence, the CP mode does not need an extra temperature sensor which is distant to the heater for measuring the original gas temperature. However, excessive temperatures might be reached at the heater when there is low or no flow through the sensor, and consequently, they may cause discomfort to the patient if the flow sensor is placed close to human tissue.

In the simulations for the CT mode, a wide range of power $(2.15 \sim 12.4 \mathrm{~W}$ ) is needed to keep the temperature constant (Fig. 2c). Hence electronics with high power rating are needed, increasing the dynamic range and physical size of the feedback circuits. In addition, Fig. $2 \mathrm{~d}$ shows that for different $T_{\infty}$, the output $\Delta T$ for a given distance $D$, is not constant. As a result, an inlet temperature sensor is necessary for the correct velocity prediction, further increasing the complexity of the feedback circuitry. This is why most of the existing sensors [12] that operate in the CT mode have stable inlet gas temperature for eliminating the need for inlet temperature compensation. For a given inlet air temperature, a higher heater temperature results in better sensitivity to the flow at the expense of higher power consumption.

The CTD operates in a similar manner to the CT, where the inlet temperature must be known in order to regulate the heater's temperature to the correct overtemperature. It also requires the monitoring of inlet temperature for the correct flow measurement. According to Fig. $2 \mathrm{f}$, the output of the CTD, i.e., $\Delta T$, has the least sensitivity to flows among the three modes.
Hence, the CTD mode requires high-resolution temperature sensors for distinguishing small changes of flow rates. However, a significant advantage of the CTD is that it requires least power among the three modes.

All three modes require a certain mechanism of feedback. The CP mode needs to monitor the voltage and current to the heater simultaneously and then regulates the product of the voltage and current, while the CT and CTD modes need to regulate the heater temperature and heater-inlet temperature difference, respectively. A precise flow measurement will rely on a speedy response from the heater to different velocities which very much depends upon the thermal time constant of the heater. Hence, minimizing the sensor size and keeping the power low help to improve the measurement accuracy and transient response.

The simulation results also validate (5) for all the modes as:

$$
\frac{P}{\left(T_{\text {hot }}-T_{\infty}\right)}=0.093+0.24 \sqrt{U}
$$

\section{B. Optimization of Heater to Temperature Sensor Distance}

For each mode, each velocity corresponds to a temperature difference between the two temperature sensors. This difference, i.e., $\Delta T$, for the same velocity, alters as the point of measurement moves away or closer to the heater. We prefer to focus on 0.25 and $1.25 \mathrm{~m} / \mathrm{s}$ velocity values since they include the most important volume rate range of human respiration while the velocity is relatively linearly related to the flow sensor's output. For each mode separately, the goal is to find the distance from the heater where the absolute difference between the $\Delta T_{1}\left(D, U_{\infty}\right.$ $=1.25)$, and $\Delta T_{2}\left(D, U_{\infty}=0.25\right)$ is the greatest, hence, to increase output range and sensitivity. The reason we are looking for the spot of the greatest difference in the outputs is that for a predefined number of resolution steps, the size of measurement step increases as the difference becomes greater, simplifying the temperature sensor design to a certain extent. Consider 30 steps between the 0.25 and $1.25 \mathrm{~m} / \mathrm{s}$ or, a resolution of approximately $0.033 \mathrm{~m} / \mathrm{s}$. By placing the temperature sensors $1.8 \mathrm{~mm}$ far from the heater while using the CTD mode, the necessary resolution for the temperature sensors will be $0.8 / 30=0.027{ }^{\circ} \mathrm{C}$ (the 0.8 value is derived by the CTD plot of Fig. 3a at $1.8 \mathrm{~mm}$ ). However, if the temperature sensors are placed $0.3 \mathrm{~mm}$ away from the heater, the resolution becomes $0.4 / 30=0.013{ }^{\circ} \mathrm{C}$, making the temperature sensor design more challenging, requiring a temperature sensor with a finer resolution for the same velocity resolution $(0.033 \mathrm{~m} / \mathrm{s})$. Also, Figs. $2 \mathrm{~d}$ and $2 \mathrm{f}$ suggest that for the CT and CTD modes there is a certain distance $D_{\mathrm{x}}$ where the output $\Delta T\left(D_{\mathrm{x}}, U_{\infty}\right)$ is relatively constant regardless of the $U_{\infty}$. It is that point in Fig. 3a where the output difference is zero. Evidently, $D_{\mathrm{x}}$ must be avoided as the position to place the temperature sensors since the flow sensor becomes rather insensitive to velocity variations. Figs. $2 \mathrm{~d}$ and $2 \mathrm{f}$ also show that for $D<D_{\mathrm{x}}$ the output increases as the flow increases, while for $D>D_{\mathrm{x}}$, it decreases for an increasing velocity. Hence, if large $D$ is not an issue, larger than $D_{\mathrm{x}}$ distances can be chosen if one is more interested in the lower end of the velocity range. In addition, by comparing the two CT curves in Fig. 3a, it is evident that for a given temperature sensor with known measurement accuracy, it can measure low-temperature flows more accurately than high-temperature flows. 

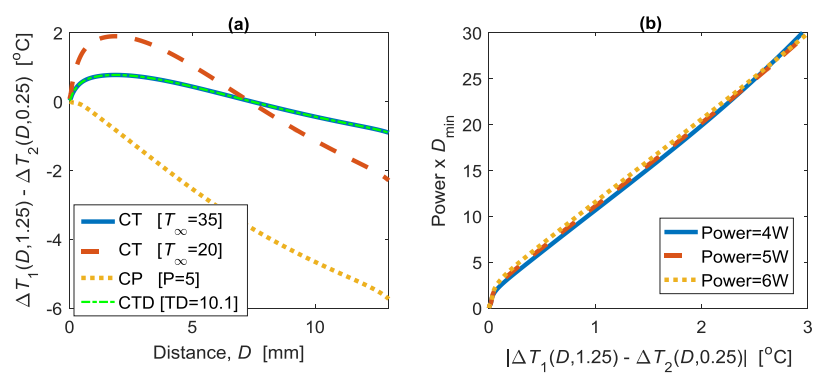

Figure 3. (a) Output difference for 0.25 to $1.25 \mathrm{~m} / \mathrm{s}$, for all distances away from the heater. The " $C T\left[T_{\infty}=35\right]$ " overlaps with the " $C T D$ ". (b) $\bar{P} \times D_{\min }$ vs. output difference for different power consumptions in the $\mathrm{CP}$ mode.

The simulation suggests that for the $\mathrm{CP}$ mode, the temperature sensors should be placed far from the heater for the highest $\left|\Delta T_{1}-\Delta T_{2}\right|$. In previous studies, the heater power was usually decided according to the predefined temperature sensor's resolution and pre-defined temperature sensor locations. However, this is an optimization process towards only the power. The simulation results of this work suggest that the necessary temperature sensor resolution is affected by both the heater power and temperature sensor-heater distance which should be optimized together. The simulation results in Fig. 3a suggest that one can get a 30 -step of $0.02{ }^{\circ} \mathrm{C}$ resolution between $0.25 \mathrm{~m} / \mathrm{s}$ and $1.25 \mathrm{~m} / \mathrm{s}$ (i.e., $\Delta T_{1}(D, 1.25)-\Delta T_{2}(D, 0.25)=30 \times$ $0.02=0.6{ }^{\circ} \mathrm{C}$ ) using either the $\mathrm{CTD}$, the $\mathrm{CP}$ or the $\mathrm{CT}$ mode and by placing the temperature sensors at $0.6,1.45$ and $0.6 \mathrm{~mm}$ respectively (for the CT the case of $35^{\circ} \mathrm{C}$ inlet temperature is considered). To have a single parameter which allows us to optimize power and size at the same time, a figure of merit is proposed which is defined as follows:

$$
\mathrm{FoM}=D_{\min } \times \bar{P}
$$

where $D_{\text {min }}$ is the minimum distance needed to achieve the necessary resolution, and $\bar{P}$ is the average value of heater's power over the inlet temperature and velocity range of interest. For both, CT and CTD mode, $\bar{P}$ can be calculated using (7) and the mean value theorem of integrals, thus:

$$
\begin{gathered}
\bar{P}=\frac{1}{U_{B}-U_{A}} \int_{U_{A}}^{U_{B}} P d U \\
\bar{P}=\frac{1}{T_{B}-T_{A}} \frac{1}{U_{B}-U_{A}} \int_{T_{A}}^{T_{B}} \int_{U_{A}}^{U_{B}} P d U d T_{\infty}
\end{gathered}
$$

where (9) refers to CTD and (10) to CT mode. $U_{\mathrm{A}}, U_{\mathrm{B}}, T_{\mathrm{A}}$ and $T_{\mathrm{B}}$ are equal to $0.25 \mathrm{~m} / \mathrm{s}, 1.25 \mathrm{~m} / \mathrm{s}, 20^{\circ} \mathrm{C}$ and $35^{\circ} \mathrm{C}$, respectively. Table II shows that for $0.033 \mathrm{~m} / \mathrm{s}\left(0.02{ }^{\circ} \mathrm{C}\right)$ resolution, the $\bar{P}$ for the CP, CT, and CTD is 5, 5.22 and $2.996 \mathrm{~W}$, respectively. The $D_{\min }$ in Table II is extracted from Fig. 3a. Among the three different modes, the CTD has the lowest FoM for the given velocity range and measurement resolution. It results in significant power and size reduction at the expense of the incoming fluid's temperature monitoring. The same analysis and FoM calculation can be applied to any other velocity range or

TABLE II. FOM RESUlTS FOR THE THREE MODES

\begin{tabular}{|c|c|c|c|}
\hline Mode & $\boldsymbol{D}_{\min }[\boldsymbol{m m}]$ & $\overline{\boldsymbol{P}}[\boldsymbol{W}]$ & FoM $[\boldsymbol{m} \boldsymbol{m} \times \boldsymbol{W}]$ \\
\hline $\mathrm{CP}$ & 1.45 & 5.000 & 7.25 \\
\hline $\mathrm{CT}$ & 0.60 & 5.220 & 3.13 \\
\hline $\mathrm{CTD}$ & 0.60 & 2.996 & 1.80 \\
\hline
\end{tabular}

experimental setup, providing a generic methodology for the optimization of calorimetric flow sensors. It can also be applied on a single mode. Since CP plot in Fig. 3a is monotonic for the distance range presented, the distance $D$ corresponds to the $D_{\text {min }}$ for the relevant output difference. Fig. $3 b$ shows the product of $\bar{P}$ and $D_{\text {min }}$ achieved over a range of output difference for different power values in the $\mathrm{CP}$ mode. The very similar product suggests that an increase in the heater power means the sensor size, which is described by $D_{\min }$, can be decreased by the same factor and vice versa. Hence, it indicates the interplay and the equal importance of $P$ and $D_{\min }$ and the fact that they should not be considered as two independent factors to optimize.

\section{CONCLUSION}

A model for the optimization of medical calorimetric flow sensors for respiratory monitoring applications to facilitate the design of temperature readout circuits, such as ADCs, has been developed. A novel Figure of Merit has been proposed, greatly simplifying the multi-dimensional optimization process in the design of calorimetric flow sensors. The performance of operating the heater in the CP, CT and CTD is compared. The simulation results suggest the CTD mode offers the best FoM for the given velocity range and measurement accuracy. Although the analysis and simulation in the paper were based on designs in the mm scale, the same principle and optimization process can be applied to any thermal flow sensor, including integrated sensor in sub-mm scale.

\section{REFERENCES}

G. Bonmarchand, et al.,"Increased initial flow rate reduces inspiratory work of breathing during pressure support ventilation in patients with exacerbation of chronic obstructive pulmonary disease," Intensive Care Med., vol. 22, pp. 1147-1154, 1996.

[2] M. R. Hetzel and T. J. Clark, "Comparison of normal and asthmatic circadian rhythms in peak expiratory flow rate.," Thorax, vol. 35, pp. 732-738, 1980

[3] M. Piotto, et al., "Smart flow sensor with on-chip CMOS interface performing offset and pressure effect compensation," IEEE Sens. J., vol. 12, no. 12, pp. 3309-3317, 2012.

[4] A. Al-Salaymeh, J. Jovanović, and F. Durst, "Bi-directional flow sensor with a wide dynamic range for medical applications," Med. Eng. Phys., vol. 26, no. 8, pp. 623-37, Oct. 2004.

[5] S. Silvestri and E. Schena, "Micromachined flow sensors in biomedical applications," Micromachines, vol. 3, pp. 225-243, 2012.

[6] F. Hedrich, et al., "Thermal flow sensors for MEMS spirometric devices," in Sensors and Actuators, A: Physical, 2010, vol. 162, no. 2, pp. 373-378.

[7] B. W. van Oudheusden, "Silicon thermal flow sensors," Sensors Actuators A Phys., vol. 30, no. 1-2, pp. 5-26, Jan. 1992.

[8] J. Sun, M. Qin, and Q. Huang, "Flip-chip packaging for a twodimensional thermal flow sensor using a copper pillar bump technology," Sensors Journal, IEEE, vol. 7, no. 1, pp. 990-995, 2007.

[9] G. Kaltsas, et al., "A smart flow measurement system for flow evaluation with multiple signals in different operation modes," Meas. Sci. Technol., vol. 18, p. 3617, 2007.

[10] L. V. King, "On the Convection of Heat from Small Cylinders in a Stream of Fluid: Determination of the Convection Constants of Small Platinum Wires, with Applications to Hot-Wire Anemometry," Proc. R. Soc. London A Math. Phys. Eng. Sci., vol. 90, no. 622, pp. 563570, 1914.

[11] W. T. Blows, The Biological Basis of Nursing: Clinical Observations. London: Routledge, 2001.

[12] N. Sabaté, et al., "Multi-range silicon micromachined flow sensor," Sensors Actuators, A Phys., vol. 110, pp. 282-288, 2004. 